\title{
Lista recenzentów \\ „Ruchu Prawniczego, Ekonomicznego i Socjologicznego" \\ za rok 2013
}

Józef Baniak

Ryszard Barczyk

Roman Budzinowski

Wojciech Dajczak

Przemysław Deszczyński

Małgorzata Doman

Rafał Drozdowski

Tadeusz Gadkowski

Andrzej Gomułowicz

Joanna Haberko

Janina Harasim

Małgorzata M. Hybka

Wacław Jarmołowicz

Andrzej Jarocha

Baha Kalinowska-Sufinowicz

Zdzisław Kędzia

Marian Kępiński

Bożena Klimczak

Krzysztof Knoppek

Tadeusz Kowalski

Marek Krajewski

Danuta Krzemińska

Wojciech Łączkowski

Ewa Małuszyńska

Kamilla Marchewka-Bartkowiak

Monika Marcinkowska

Dominik Mączyński

Jacek Napierała

Zdzisław Niedbała
Agnieszka Niezgoda

Aurelia Nowicka

Hanna Paluszkiewicz

Janina Panowicz-Lipska

Krzysztof Podemski

Łukasz Pohl

Wiesława Przybylska-Kapuścińska

Irena Pyka

Marek Ratajczak

Jan Sandorski

Michał Skapski

Marek Smolak

Tomasz Sokołowski

Andrzej J. Szwarc

Andrzej Tobis

Anna Trela

Jan Tyranowski

Elżbieta Urbanowska-Sojkin

Andrzej Wasilewski

Paweł Wiliński

Jan Winiecki

Jan Włodarek

Krystyna Wojtczak

Zbigniew Woźniak

Robert Zawłocki

Maciej Zieliński

Maria Zmierczak

Aldona Żurek 